\title{
Further Study on MOE-Based Multiuser Detection in Unknown Multipath
}

\author{
Zhengyuan Xu \\ Department of Electrical Engineering, University of California, Riverside, CA 92521, USA \\ Email:dxu@ee.ucr.edu
}

Received 9 January 2002

\begin{abstract}
The minimum output energy (MOE) receiver has been developed for multiuser detection when multipath distortion is present. Its performance has been shown to be very close to the minimum mean square error (MMSE) receiver at high signal-to-noise ratio. However, due to the additive noise, the constraint vector required to construct the MOE receiver is a biased estimate of the channel vector. Thus, the MOE receiver exhibits degraded performance. To mitigate the noise effect, the constraint cost function is modified to obtain a modified MOE (MMOE) receiver in this paper, leading to a significantly improved channel estimate and detection performance. It is also revealed that the MMOE method converges to the well-known subspace method under certain conditions. In addition to the additive noise, imperfect estimation of the output data covariance matrix also causes performance loss and it is studied in details based on perturbation theory.
\end{abstract}

Keywords and phrases: minimum output energy, constrained optimization, perturbation analysis.

\section{INTRODUCTION}

The rapidly growing demands for integrated wideband/ broadband services have created considerable research interest in developing new wireless communication technologies $[1,2]$. Among several options for the implementation of the air interface of future wideband wireless systems, direct sequence (DS) code division multiple access (CDMA) spread spectrum has emerged as a leading technology due to its intriguing features. It allows many users to simultaneously share the finite amount of available spectrum with minimal coordination, thus increase the system capacity. It also exhibits exceptional robustness to jamming, interception and multipath fading. Additionally, it provides much flexibility in system design and implementation by its unique spreading mechanism. This technology will continue to thrive in multiuser systems and will further establish its dominance in the future wideband wireless networks.

In a DS/CDMA system, multiuser interference (MUI) is a typical obstacle to be obviated in detection of input signals. Substantial efforts have focused on multiuser detection $[3,4,5,6]$. Although the optimal receiver is well recognized to be the maximum likelihood sequence estimator (MLSE) [7], linear detectors receive considerable attention. Their low complexity, ease of implementation, and acceptable performance make them so attractive in many applications. Among those linear detectors, blind solutions are particularly suitable for a bandwidth constrained system. Without multipath fading, a blind minimum mean square error (MMSE) detector can be implemented under certain constraints [8]. If multipath propagation occurs, a direct blind MMSE receiver has been reported [9] which requires a noise subspace from the eigenvalue decomposition (EVD) of a data covariance matrix. Subspace methods not only provide sufficiently good channel estimates even for a short data record, but also yield MMSE detectors $[10,11,12,13]$.

Constrained minimum output energy (MOE) approaches $[14,15]$ and subsequently developed robust methods $[16,17]$ and provide direct detection techniques in the presence of unknown multipath distortions. These blind MOE methods minimize the output energy (or power) of the receiver subject to certain constraints to guarantee no cancellation of the desired signal. Constraints can be parameterized and optimized in order to optimally combine signals from different paths. The MOE receiver proposed in [15] approaches the blind MMSE receiver at high signal-to-noise ratio (SNR), making it an attractive blind solution. However, due to the presence of additive white Gaussian noise (AWGN), the constraint vector has been shown to be a biased estimate of the channel vector. The bias, in terms of the vector norm, is proportional to the noise power. Thus, some performance loss from the MOE receiver is induced when compared with the MMSE receiver. Detailed study on the noise effect has been performed in [15].

In order to mitigate the noise effect and obtain an unbiased channel estimate, we modify the constrained cost function adopted in [15] by removing the contribution of the noise (parameterized by the noise power) to the data 
covariance matrix. Following the procedure of constrained MOE optimization, a new solution is similarly derived. It is shown that the resulting modified MOE (MMOE) receiver significantly outperforms the MOE receiver by properly selecting an adjusting parameter pertaining to the noise power. Meanwhile, if the MMOE method is applied to estimate the channel parameters, it is closely related to the subspace-based channel estimation method, for example, [10]. Under certain conditions on the choice of the parameter, it will be revealed that these two methods employ approximately the same cost function, leading to a convergent solution. In such a case, the MMOE receiver also coincides with the MMSE receiver.

Besides the additive noise, imperfect estimation of the output data covariance matrix based on a finite number of data samples also degrades the detection performance. Some preliminary results have been reported in [18]. In practice, only finite number $(N)$ of snapshots are collected for processing. Since the MOE receiver employs the second order statistics of the channel output which are estimated from the received data, the accuracy of covariance estimation depends on the available data record. Due to this estimation error, the optimal constraint vector is perturbed to be dependent on $N$. It will finally convey an error to the receiver's parameters and result in performance loss of MOE-based detection. Although some simulation results are provided in [15], lack of analytical results also motivates us to further study the effect of $N$ on the performance of the MOE method in detail in this paper.

It is easily observed that perturbation exists in the estimated data covariance matrix. This quantity is essential for the behavior of the constrained cost function optimized in the MOE method. Once the objective matrix in the cost function gets perturbed, one of its eigenvectors, which is the optimal constraint vector, is perturbed. Hence, the performance of the receiver will degrade for a finite $N$. Based on perturbation analysis $[19,20]$ and the analytical expression of the MOE receiver, the performance of the perturbed receiver, in terms of the output signal-to-interference-plus-noise ratio (SINR), can be evaluated for different $N$ and further compared with that of the ideal MOE receiver. Our analytical results are of much theoretical importance in predicting the system performance for any given set of system parameters. All those results are verified by computer simulations.

The paper is organized as follows. Section 2 presents a DS/CDMA system to be studied. After a brief review of the MOE multiuser detection method in Section 3, our modified MOE method is proposed and analyzed in Section 4 by taking into account the noise effect. Effect of finite data samples is investigated in Section 5. Numerical examples are then provided in Section 6 to justify our analyses and conclusions are drawn in the last section.

\section{SYSTEM MODEL}

Consider a DS/CDMA communication system with $J$ users. User $j(j=1, \ldots, J)$ has a zero-mean and i.i.d. information, bearing sequence $w_{j}(n)$ to transmit whose variance is $\sigma_{w_{j}}^{2}=$ $E\left\{\left\|w_{j}(n)\right\|^{2}\right\}$. Each symbol is spread by a periodic spreading sequence $c_{j}(k)(k=0, \ldots, P-1)$ of period $P$. Then the signal after spreading is given in [15]

$$
s_{j}(n)=\sum_{l=-\infty}^{\infty} w_{j}(l) c_{j}(n-l P)
$$

Let the chip sequence be transmitted through a linear channel with a baseband impulse response $g_{j}(n)$. Then the received discrete-time signal $y(n)$ at the chip rate receiver due to user $j$ becomes

$$
\begin{aligned}
y_{j}(n) & =\sum_{m=-\infty}^{\infty} g_{j}(m) s_{j}\left(n-d_{j}-m\right) \\
& =\sum_{l=-\infty}^{\infty} w_{j}(l) h_{j}\left(n-d_{j}-l P\right),
\end{aligned}
$$

where $d_{j}$ is the delay of user $j$ in chip periods,

$$
h_{j}(n)=\sum_{m=-\infty}^{\infty} g_{j}(m) c_{j}(n-m) .
$$

After considering all users and AWGN with zero-mean and variance $\sigma_{v}^{2}=E\left\{\|v(n)\|^{2}\right\}$, the received signal becomes

$$
y(n)=\sum_{j=1}^{J} y_{j}(n)+v(n) .
$$

Assume $0 \leq d_{j}<P$ and the receiver is synchronized to our desired user-user $1\left(d_{1}=0\right)$. The channel $g_{j}(n)$ has maximum order $q \ll P$ [15]. After collecting $P$ measurements in a vector, a vector form input/output relation follows

$$
\mathbf{y}(n)=\sum_{j=1}^{J} \mathbf{y}_{j}(n)=\mathbf{h}_{1} w_{1}(n)+\mathbf{H}_{i} \mathbf{w}_{i}(n)+\mathbf{v}(n),
$$

where $\mathbf{h}_{1}=\left[h_{1}(0), \ldots, h_{1}(P-1)\right]^{T}$ is the signature vector of user $1, \mathbf{w}_{i}(n)$ is an interference vector including intersymbol interference (ISI) and MUI, $\mathbf{H}_{i}$ is the signature matrix with columns representing signature waveforms of corresponding symbols in $\mathbf{w}_{i}(n), \mathbf{v}(n)$ is an AWGN vector. Based on (3), the signature vector of $w_{1}(n)$ can be decomposed as $\mathbf{h}_{1}=\mathbf{C g}_{1}$ where $\mathbf{C}$ is a code filtering matrix corresponding to the desired user and $\mathbf{g}_{1}$ is its unknown channel vector

$$
\begin{aligned}
\mathbf{C} & =\left[\begin{array}{ccc}
c_{1}(0) & & 0 \\
\vdots & \ddots & c_{1}(0) \\
\vdots & & \vdots \\
c_{1}(P-1) & \cdots & c_{1}(P-q-1)
\end{array}\right], \\
\mathbf{g}_{1} & =\left[\begin{array}{c}
g_{1}(0) \\
\vdots \\
g_{1}(q)
\end{array}\right] .
\end{aligned}
$$

This structure of the user's signature has been exploited in [15] to design an MOE receiver which will be first briefly reiterated next. 


\section{MOE-BASED MULTIUSER DETECTION}

When multipath distortion exists in a communication system, an MOE receiver $f$ is obtained by minimizing its output power subject to parameterized constraints $\mathbf{g}$ as [15]

$$
\min _{\mathbf{f}} \mathbf{f}^{H} \mathbf{R f}, \quad \text { subject to } \mathbf{C}^{H} \mathbf{f}=\mathbf{g},
$$

where $\mathbf{R}=E\left\{\mathbf{y}(n) \mathbf{y}^{H}(n)\right\}$. In (7), $\mathbf{g}$ is a constraint vector containing $q+1$ unknown constraints. Under multiple constraints, the power of the desired symbol becomes a constant. Therefore, minimization of the output power is equivalent to minimization of interference plus noise. For a given $\mathbf{g}$, the optimal receiver can be derived, based on the Lagrange multiplier method

$$
\mathbf{f}_{0}=\mathbf{R}^{-1} \mathbf{C}\left(\mathbf{C}^{H} \mathbf{R}^{-1} \mathbf{C}\right)^{-1} \mathbf{g}
$$

while the minimum output power becomes

$$
\Phi_{\min }=\mathbf{g}^{H}\left(\mathbf{C}^{H} \mathbf{R}^{-1} \mathbf{C}\right)^{-1} \mathbf{g} .
$$

In order to obtain an optimal constraint vector, it is proposed to maximize $\mathscr{J}_{\min }[15]$, that is, to maximize the power of the signal component after the interference has been suppressed in the first step. It is then transformed into the following problem:

$$
\mathbf{g}_{0}=\arg \min _{\|\mathbf{g}\|=1} \mathbf{g}^{H} \mathbf{A g}, \quad \mathbf{A} \triangleq \mathbf{C}^{H} \mathbf{R}^{-1} \mathbf{C}
$$

Therefore, $\mathbf{g}_{0}$ is the eigenvector of $\mathbf{A}$ associated with its minimum eigenvalue $\lambda$. With $\mathbf{g}_{0}$, the MOE receiver in (8) irrespective of a positive scalar $1 / \lambda$ becomes

$$
\mathbf{f}_{0}=\mathbf{R}^{-1} \mathbf{C g}_{0}
$$

It can be observed from (11) that if $\mathbf{g}_{0}$ is replaced by $\mathbf{g}_{1}$, then $\mathbf{f}_{0}$ becomes the MMSE solution after ignoring a positive scalar. Unfortunately, such an MOE receiver exhibits near-optimal performance [15] compared with the MMSE receiver mainly due to the fact that $\mathbf{g}_{0}$ is a biased estimate of $\mathbf{g}_{1}$. As revealed therein, the first-order discrepancy between $\mathbf{g}_{0}$ and $\mathbf{g}_{1}$ is proportional to the noise power $\sigma_{v}^{2}$. If the noise power is reduced in the output covariance matrix, the estimate of $\mathbf{g}_{1}$ is expected to be improved. Finally, the detection performance will approach that of the MMSE receiver. In Section 4, we will mitigate this noise effect by modifying the optimization cost function and develop a corresponding criterion.

\section{MITIGATION OF NOISE IN MOE-BASED MULTIUSER DETECTION}

\subsection{Modified optimization criterion}

The contribution of noise exists in the data covariance matrix $\mathbf{R}$. We reconstruct a cost function by alleviating the noise contribution $^{1}$

$$
\min _{\overline{\boldsymbol{\theta}}} \overline{\boldsymbol{\theta}}^{H} \overline{\mathbf{R}} \overline{\boldsymbol{\theta}}, \quad \text { subject to } \mathbf{C}^{H} \overline{\boldsymbol{\theta}}=\overline{\mathbf{g}},
$$

where $\overline{\mathbf{R}}=\mathbf{R}-\alpha \sigma_{v}^{2} \mathbf{I}$. Parameter $\alpha$ quantifies the extent of noise mitigation and should satisfy $0 \leq \alpha<1$ such that the modified covariance matrix $\overline{\mathbf{R}}$ is positive definite. Its effect will be discussed later. Vector $\overline{\boldsymbol{\theta}}$ is a pretended receiver because it filters the denoised data which is difficult to obtain in practice. The main objective in this step is to formulate an optimization problem which guarantees a better solution for our channel estimate, upon which a practical MMSE receiver can be built. The optimal "receiver" from (12) becomes

$$
\overline{\boldsymbol{\theta}}_{0}=\overline{\mathbf{R}}^{-1} \mathbf{C}\left(\mathbf{C}^{H} \overline{\mathbf{R}}^{-1} \mathbf{C}\right)^{-1} \overline{\mathbf{g}},
$$

while the minimum output power has a form

$$
\mathscr{F}_{\min }=\overline{\mathbf{g}}^{H}\left(\mathbf{C}^{H} \overline{\mathbf{R}}^{-1} \mathbf{C}\right)^{-1} \overline{\mathbf{g}} .
$$

Following the similar procedure described in Section 3, $\mathbf{\mathbf { g }}$ is obtained by

$$
\overline{\mathbf{g}}_{0}=\arg \min _{\|\overline{\mathbf{g}}\|=1} \overline{\mathbf{g}}^{H} \overline{\mathbf{A}} \overline{\mathbf{g}}, \quad \overline{\mathbf{A}} \triangleq \mathbf{C}^{H} \overline{\mathbf{R}}^{-1} \mathbf{C} .
$$

Once optimal solution $\overline{\mathbf{g}}_{0}$ is obtained, our MMOE receiver is forced to take a similar form as (11)

$$
\overline{\mathbf{f}}_{0}=\mathbf{R}^{-1} \mathbf{C} \overline{\mathbf{g}}_{0}
$$

instead of being constructed from $\overline{\mathbf{R}}$. However, it is noticed that both $\overline{\mathbf{R}}$ and $\mathbf{R}$ are required to obtain $\overline{\mathbf{f}}_{0}$ since $\overline{\mathbf{R}}$ affects $\overline{\mathbf{g}}_{0}$. In practice, $\mathbf{R}$ can be easily estimated from the received data vectors. But the noise power is not known a priori, rendering estimation of $\overline{\mathbf{R}}$ more difficult. We may proceed to obtain an estimate of $\sigma_{v}^{2}$ from the smallest eigenvalue of the estimated data covariance matrix $\widetilde{\mathbf{R}}$. Then choose $\alpha$ as close to one as possible but ensure that $\overline{\mathbf{R}}$ does not become rank deficient. It may also be feasible to treat $\alpha \sigma_{v}^{2}$ as a new parameter and preselect its value based on some preliminary knowledge about the noise level in a practical system.

Next we will study how parameter $\alpha$ affects our channel estimator $\overline{\mathbf{g}}_{0}$ and the performance of the receiver $\overline{\mathbf{f}}_{0}$. In particular, the channel estimation error and the output SINR of the MMOE receiver will be derived and compared with the MOE-based approach.

\subsection{Performance of the MMOE method}

According to (15), (16), and our definition for $\overline{\mathbf{R}}$, the performance of the modified channel estimator (constraint vector) and the MMOE receiver are highly dependent on the choice of $\alpha$. For notational convenience, define $\beta=(1-\alpha) \sigma_{v}^{2}$. A procedure similar to [15] will be taken in the following analysis. First, under the assumption that $\beta$ is small, we express the optimal constraint vector as a power series of $\beta$. Thus, the

\footnotetext{
${ }^{1}$ Quantities with “_” above are for the modified method.
} 
channel estimation error can be compared with [15]. It will be revealed that the error is significantly reduced. Then we will derive the SINR as a function of $\alpha$ and show that SINR is also improved due to more accurate channel estimate. $\overline{\mathbf{R}}[15]$

To achieve our goal, we similarly perform EVD on $\mathbf{R}$ and

$$
\begin{aligned}
& \mathbf{R}=\left[\begin{array}{ll}
\mathbf{V}_{s} & \mathbf{V}_{n}
\end{array}\right]\left[\begin{array}{cc}
\boldsymbol{\Lambda}_{s} & \mathbf{0} \\
\mathbf{0} & \mathbf{0}
\end{array}\right]\left[\begin{array}{c}
\mathbf{V}_{s}^{H} \\
\mathbf{V}_{n}^{H}
\end{array}\right]+\sigma_{v}^{2} \mathbf{I}, \\
& \overline{\mathbf{R}}=\left[\begin{array}{ll}
\mathbf{V}_{s} & \mathbf{V}_{n}
\end{array}\right]\left[\begin{array}{cc}
\boldsymbol{\Lambda}_{s} & \mathbf{0} \\
\mathbf{0} & \mathbf{0}
\end{array}\right]\left[\begin{array}{c}
\mathbf{V}_{s}^{H} \\
\mathbf{V}_{n}^{H}
\end{array}\right]+\beta \mathbf{I},
\end{aligned}
$$

where $\boldsymbol{\Lambda}_{s}=\operatorname{diag}\left\{\lambda_{1}, \ldots, \lambda_{\xi}\right\}, \mathbf{V}_{s}$, and $\mathbf{V}_{n}$ span the signal and noise subspaces, respectively. ${ }^{2}$ In parallel with [15, Lemmas 1 and 2], we first present the following results. For simplicity, assume $\left\|\mathbf{g}_{1}\right\|=1$.

Lemma 1. The objective matrix $\overline{\mathbf{A}}$ in (15) after being scaled by $\beta$ can be expressed by a power series of $\beta$, similarly as matrix $\mathbf{A}$ by a power series of $\sigma_{v}^{2}$,

$$
\begin{aligned}
\beta \mathbf{C}^{H} \overline{\mathbf{R}}^{-1} \mathbf{C} & =\mathbf{A}_{0}+\beta \mathbf{A}_{1}-\beta^{2} \mathbf{A}_{2}+\mathbf{O}\left(\beta^{3}\right), \\
\sigma_{v}^{2} \mathbf{C}^{H} \mathbf{R}^{-1} \mathbf{C} & =\mathbf{A}_{0}+\sigma_{v}^{2} \mathbf{A}_{1}-\sigma_{v}^{4} \mathbf{A}_{2}+\mathbf{O}\left(\sigma_{v}^{6}\right),
\end{aligned}
$$

where matrices $\mathbf{A}_{0}, \mathbf{A}_{1}$, and $\mathbf{A}_{2}$ are defined as ${ }^{3}$

$$
\begin{aligned}
& \mathbf{A}_{0}=\mathbf{C}^{H} \mathbf{V}_{n} \mathbf{V}_{n}^{H} \mathbf{C}, \\
& \mathbf{A}_{1}=\mathbf{C}^{H} \mathbf{V}_{s} \boldsymbol{\Lambda}_{s}^{-1} \mathbf{V}_{s}^{H} \mathbf{C}, \\
& \mathbf{A}_{2}=\mathbf{C}^{H} \mathbf{V}_{s} \boldsymbol{\Lambda}_{s}^{-2} \mathbf{V}_{s}^{H} \mathbf{C} .
\end{aligned}
$$

Lemma 2. If $\overline{\mathbf{A}}=\mathbf{C}^{H} \overline{\mathbf{R}}^{-1} \mathbf{C}$ satisfies (18), then its minimum eigenvalue $\bar{\gamma}_{\min }$ and eigenvector $\overline{\mathbf{g}}$ are given by

$$
\begin{aligned}
\bar{\gamma}_{\min } & =\frac{1}{\sigma_{w_{1}}^{2}}-\beta \mathbf{g}_{1}^{H} \mathbf{A}_{2} \mathbf{g}_{1}-\beta \mathbf{g}_{1}^{H} \mathbf{A}_{1} \mathbf{A}_{0}^{\dagger} \mathbf{A}_{1} \mathbf{g}_{1}+O\left(\beta^{2}\right), \\
\overline{\mathbf{g}}_{0} & =\mathbf{g}_{1}-\beta \mathbf{A}_{0}^{\dagger} \mathbf{A}_{1} \mathbf{g}_{1}+\mathbf{O}\left(\beta^{2}\right) .
\end{aligned}
$$

Meanwhile, the following holds:

$$
\sigma_{w_{1}}^{2} \mathbf{g}_{1}^{H} \mathbf{A}_{1} \mathbf{g}_{1}=1
$$

where $\dagger$ denotes the pseudo-inverse.

These two lemmas can be similarly proved as in [15]. According to Lemma 2, the first-order bias in channel estimate becomes

$$
\Delta \overline{\mathbf{g}} \approx-(1-\alpha) \sigma_{v}^{2} \mathbf{A}_{0}^{\dagger} \mathbf{A}_{1} \mathbf{g}_{1}
$$

where $\beta$ has been replaced by its definition $(1-\alpha) \sigma_{v}^{2}$. Noticing the bias $\Delta \mathbf{g}$ from the MOE method

\footnotetext{
${ }^{2}$ We have dropped and will drop the subscripts for all identity matrices I which can be easily identified from the context.

${ }^{3}$ For clarity later, the matrix $\mathbf{A}_{2}$ is defined to be positive definite which is opposite to that in [15].
}

$$
\Delta \mathbf{g} \approx-\sigma_{v}^{2} \mathbf{A}_{0}^{\dagger} \mathbf{A}_{1} \mathbf{g}_{1}
$$

the channel estimation errors from the MMOE method and the MOE method are related to each other by $\|\Delta \overline{\mathbf{g}}\| \approx(1-$ $\alpha)\|\Delta \mathbf{g}\|$. Recalling that $0 \leq \alpha<1,\|\Delta \overline{\mathbf{g}}\|$ is thus reduced, such that $\|\Delta \overline{\mathbf{g}}\|<\|\Delta \mathbf{g}\|$. Especially when $\alpha$ approaches 1 (from the left) $\alpha \rightarrow 1^{-},\|\Delta \overline{\mathbf{g}}\| \ll\|\Delta \mathbf{g}\|$ and $\|\Delta \overline{\mathbf{g}}\| \rightarrow 0$.

With these results, an interesting observation can be easily made. The MMOE method is closely related to the subspace method, for example, [10]. In fact, as $\alpha \rightarrow 1^{-}, \beta \rightarrow 0$. It is known that scaling a matrix by a constant scalar does not alter eigenvectors of the matrix. According to (18), the objective matrix of the MMOE method (scaled by $\beta$ ) converges to $\mathbf{A}_{0}$ which is exactly the one used in the subspace method to estimate channel parameters. Therefore, the constraint vector can be well treated as an estimate of $\mathbf{g}_{1}$. The difference between the MMOE solution and the subspace solution diminishes as $\beta \rightarrow 0$. It is thus not surprising that the performance of the MMOE receiver improves, as shown by the following proposition.

Proposition 3. The SINRs of the MMSE receiver, the MOE receiver, and the proposed $M M O E$ receiver are given by

$$
\begin{aligned}
\operatorname{SINR}_{\text {mmse }} & =\frac{1+O\left(\sigma_{v}^{2}\right)}{\sigma_{v}^{2} \sigma_{w_{1}}^{2} \mathbf{g}_{1}^{H} \mathbf{A}_{2} \mathbf{g}_{1}+O\left(\sigma_{v}^{4}\right)} \\
\operatorname{SINR}_{\text {moe }} & =\frac{1+O\left(\sigma_{v}^{2}\right)}{\sigma_{v}^{2} \sigma_{w_{1}}^{2} \mathbf{g}_{1}^{H} \mathbf{A}_{2} \mathbf{g}_{1}+\sigma_{v}^{2} \sigma_{w_{1}}^{2} \mathbf{g}_{1}^{H} \mathbf{A}_{1} \mathbf{A}_{0}^{\dagger} \mathbf{A}_{1} \mathbf{g}_{1}+O\left(\sigma_{v}^{4}\right)},
\end{aligned}
$$

$$
\overline{\mathrm{SINR}}_{\mathrm{mmoe}}=\frac{1+O\left(\sigma_{v}^{2}\right)}{\sigma_{v}^{2} \sigma_{w_{1}}^{2} \mathbf{g}_{1}^{H} \mathbf{A}_{2} \mathbf{g}_{1}+(1-\alpha)^{2} \sigma_{v}^{2} \sigma_{w_{1}}^{2} \mathbf{g}_{1}^{H} \mathbf{A}_{1} \mathbf{A}_{0}^{\dagger} \mathbf{A}_{1} \mathbf{g}_{1}+O\left(\sigma_{v}^{4}\right)} .
$$

Proof. See Appendix A.

According to this proposition, we immediately obtain the following results in parallel with [15, Proposition 4], which measure the performance degradation of the MOE receiver and the MMOE receiver relative to the MMSE receiver, respectively

$$
\begin{aligned}
& \frac{\text { SINR }_{\text {mmse }}}{\mathrm{SINR}_{\mathrm{moe}}} \longrightarrow 1+\eta \quad \text { as } \sigma_{v}^{2} \longrightarrow 0, \\
& \frac{\mathrm{SINR}_{\mathrm{mmse}}}{\mathrm{SINR}_{\mathrm{mmoe}}} \longrightarrow 1+\bar{\eta} \quad \text { as } \sigma_{v}^{2} \longrightarrow 0,
\end{aligned}
$$

where

$$
\eta=\frac{\mathbf{g}_{1}^{H} \mathbf{A}_{1} \mathbf{A}_{0}^{\dagger} \mathbf{A}_{1} \mathbf{g}_{1}}{\mathbf{g}_{1}^{H} \mathbf{A}_{2} \mathbf{g}_{1}}, \quad \bar{\eta}=(1-\alpha)^{2} \eta .
$$

It is clear that the MMSE receiver provides the best performance because $\eta>0$ and $\bar{\eta}>0$. However, since $0 \leq \alpha<1$, we get $\bar{\eta} \leq \eta$. Therefore, if $\alpha$ is chosen to be non-zero, performance improvement of the MMOE receiver over the MOE receiver [15] is always guaranteed. The extent of improvement depends on $\alpha$ and system parameters which are 
indicated by quantities $\mathbf{g}_{1}, \mathbf{A}_{0}, \mathbf{A}_{1}$, and $\mathbf{A}_{2}$. In particular, when $\alpha$ approaches unity, $\bar{\eta} \rightarrow 0$ which indicates that the MMOE receiver approaches the MMSE receiver. These analytical results will be verified by our computer simulations.

Besides the additive noise, imperfect estimation of the data covariance matrix also causes performance degradation of the MOE receiver and will be studied in Section 5.

\section{PERFORMANCE LOSS IN MOE-BASED MULTIUSER DETECTION DUE TO IMPERFECT COVARIANCE ESTIMATION}

As observed from (10) and (11), $\mathbf{g}_{0}$ and $\mathbf{f}_{0}$ depend on $\mathbf{R}$. If it is perturbed, then $\mathbf{f}_{0}$ gets perturbed. We will denote a perturbation by preceding the corresponding quantity by $\delta$, and perturbed quantity with ${ }^{\sim}$

$$
\delta \mathbf{f}_{0}=\widetilde{\mathbf{f}}_{0}-\mathbf{f}_{0}, \quad \delta \mathbf{R}=\widetilde{\mathbf{R}}-\mathbf{R} .
$$

In practice, $\mathbf{R}$ is estimated by sample average $\widetilde{\mathbf{R}}=$ $(1 / N) \sum_{n=1}^{N} \mathbf{y}(n) \mathbf{y}^{H}(n)$. Perturbation arises in the estimated data covariance matrix when it is estimated from $N$ data vectors [19]. Although $\widetilde{\mathbf{R}}$ converges to $\mathbf{R}$ as $N \rightarrow \infty$, a perturbation $\delta \mathbf{R}$ due to finite $N$ will cause matrix A perturbed and finally the MOE solution. Matrix $\delta \mathbf{R}$ is a random variable due to randomness of $\widetilde{\mathbf{R}}$. It has zero-mean $E\{\delta \mathbf{R}\}=\mathbf{0}$. Here, we are interested in a perturbed eigenvector of $\mathbf{A}$ after perturbation in $\mathbf{R}$, and investigate how $N$ affects the MSE of the estimate of the constraint vector and the performance of the MOE receiver.

According to (10), A depends on $\mathbf{R}^{-1}$. Under small perturbation assumption (large $N$ ) and using Taylor's expansion up to the first order, $(\mathbf{R}+\delta \mathbf{R})^{-1}$ is approximated by

$$
(\mathbf{R}+\delta \mathbf{R})^{-1} \approx \mathbf{R}^{-1}-\mathbf{R}^{-1} \delta \mathbf{R} \mathbf{R}^{-1} .
$$

Then the first-order perturbation of $\mathbf{A}$ is

$$
\delta \mathbf{A} \approx-\mathbf{C}^{H} \mathbf{R}^{-1} \delta \mathbf{R} \mathbf{R}^{-1} \mathbf{C} .
$$

Due to $\delta \mathbf{A}, \mathbf{g}_{0}$ and associated eigenvalue $\lambda$ are perturbed with perturbations $\delta \mathbf{g}_{0}$ and $\delta \lambda$, respectively. It can be found that [19]

$$
\delta \mathbf{g}_{0} \approx-(\mathbf{A}-\lambda \mathbf{I})^{\dagger} \delta \mathbf{A g}_{0} .
$$

Substituting (33) in (34), $\delta \mathbf{g}_{0}$ is related to $\delta \mathbf{R}$ by

$$
\delta \mathbf{g}_{0} \approx(\mathbf{A}-\lambda \mathbf{I})^{\dagger} \mathbf{C}^{H} \mathbf{R}^{-1} \delta \mathbf{R} \mathbf{f}_{0},
$$

where (11) has been used. Therefore, the covariance of channel estimate becomes

$$
E\left\{\delta \mathbf{g}_{0} \delta \mathbf{g}_{0}^{H}\right\} \approx(\mathbf{A}-\lambda \mathbf{I})^{\dagger} \mathbf{C}^{H} \mathbf{R}^{-1} E\left\{\delta \mathbf{R} \mathbf{f}_{0} \mathbf{f}_{0}^{H} \delta \mathbf{R}\right\} \mathbf{R}^{-1} \mathbf{C}(\mathbf{A}-\lambda \mathbf{I})^{\dagger} .
$$

The MSE of the estimate of the constraint vector is then the trace of matrix $E\left\{\delta \mathbf{g}_{0} \delta \mathbf{g}_{0}^{H}\right\}$. Meanwhile, considering (11) and (32), the perturbation in the MOE receiver due to $\delta \mathbf{R}$ and $\delta \mathbf{g}_{0}$ has a form

$$
\delta \mathbf{f}_{0} \approx \mathbf{R}^{-1} \mathbf{C} \delta \mathbf{g}_{0}-\mathbf{R}^{-1} \delta \mathbf{R} \mathbf{f}_{0}
$$

Substituting (35) in (37), $\delta \mathbf{f}_{0}$ is related to a random variable $\delta \mathbf{R}$ by

$$
\delta \mathbf{f}_{0} \approx \mathbf{A}_{3} \delta \mathbf{R} \mathbf{f}_{0}
$$

where

$$
\mathbf{A}_{3} \triangleq\left[\mathbf{R}^{-1} \mathbf{C}(\mathbf{A}-\lambda \mathbf{I})^{\dagger} \mathbf{C}^{H}-\mathbf{I}\right] \mathbf{R}^{-1} .
$$

It also has a zero-mean $E\left\{\delta \mathbf{f}_{0}\right\}=\mathbf{0}$. The performance of the MOE receiver will be affected by $\delta \mathbf{f}_{0}$. If we adopt the SINR as a performance measure and define it as

$$
\widetilde{\mathrm{SINR}} \triangleq \sigma_{w_{1}}^{2} \frac{E\left\{\left\|\tilde{\mathbf{f}}_{0}^{H} \mathbf{h}_{1}\right\|^{2}\right\}}{E\left\{\tilde{\mathbf{f}}_{0}^{H} \mathbf{R}_{i} \tilde{\mathbf{f}}_{0}\right\}}, \quad \mathbf{R}_{i}=\mathbf{R}-\sigma_{w_{1}}^{2} \mathbf{h}_{1} \mathbf{h}_{1}^{H},
$$

then after replacing $\widetilde{\mathbf{f}}_{0}$ by $\mathbf{f}_{0}+\delta \mathbf{f}_{0}$ and noticing that $E\left\{\delta \mathbf{f}_{0}\right\}=$ $\mathbf{0}$, it is perturbed to be

$$
\widetilde{\operatorname{SINR}}=\sigma_{w_{1}}^{2} \frac{\left\|\mathbf{f}_{0}^{H} \mathbf{h}_{1}\right\|^{2}+E\left\{\delta \mathbf{f}_{0}^{H} \mathbf{h}_{1} \mathbf{h}_{1}^{H} \delta \mathbf{f}_{0}\right\}}{\mathbf{f}_{0}^{H} \mathbf{R}_{i} \mathbf{f}_{0}+E\left\{\delta \mathbf{f}_{0}^{H} \mathbf{R}_{i} \delta \mathbf{f}_{0}\right\}} .
$$

Observe that

$$
\begin{aligned}
E\left\{\delta \mathbf{f}_{0}^{H} \mathbf{h}_{1} \mathbf{h}_{1}^{H} \delta \mathbf{f}_{0}\right\} & \approx \mathbf{f}_{0}^{H} E\left\{\delta \mathbf{R} \mathbf{A}_{3}^{H} \mathbf{h}_{1} \mathbf{h}_{1}^{H} \mathbf{A}_{3} \delta \mathbf{R}\right\} \mathbf{f}_{0}, \\
E\left\{\delta \mathbf{f}_{0}^{H} \mathbf{R}_{i} \delta \mathbf{f}_{0}\right\} & \approx \mathbf{f}_{0}^{H} E\left\{\delta \mathbf{R} \mathbf{A}_{3}^{H} \mathbf{R}_{i} \mathbf{A}_{3} \delta \mathbf{R}\right\} \mathbf{f}_{0} .
\end{aligned}
$$

To evaluate these two quantities, it suffices to determine the following general-form quantity

$$
\mathbf{B} \triangleq E\{\delta \mathbf{R D} \delta \mathbf{R}\}
$$

where $\mathbf{D}$ can be replaced by $\mathbf{A}_{3}^{H} \mathbf{h}_{1} \mathbf{h}_{1}^{H} \mathbf{A}_{3}$ or $\mathbf{A}_{3}^{H} \mathbf{R}_{i} \mathbf{A}_{3}$. Similarly, if $\mathbf{D}$ is replaced by $\mathbf{f}_{0} \mathbf{f}_{0}^{H}$, then (36) can be evaluated as well. Matrix B depends on the second-order statistics of $\delta \mathbf{R}$. Therefore, it will finally rely on up to the fourth-order statistics of the received data since $\delta \mathbf{R}$ is related to the secondorder information of the output. It is shown in Appendix B that for a given data model, statistical properties of the input, and additive noise, $\mathbf{B}$ can always be evaluated according to (B.20) in Appendix B.

It is clear that both the MSE and $\widetilde{\text { SINR }}$ depend on $1 / N$. They also depend on the signal and noise powers. The effect of $N$ will be tested and compared with our analytical results by simulations.

\section{SIMULATIONS}

We simulate a 10-user CDMA system with equal power and binary inputs. Gold sequence of length 31 is used for each user. The receiver is synchronized to user 1 while other delays are uniformly distributed in $[0,30]$. Channel order is set to be 3 for each user. Channel coefficients are randomly selected from the interval $[-1,+1]$ and the corresponding 


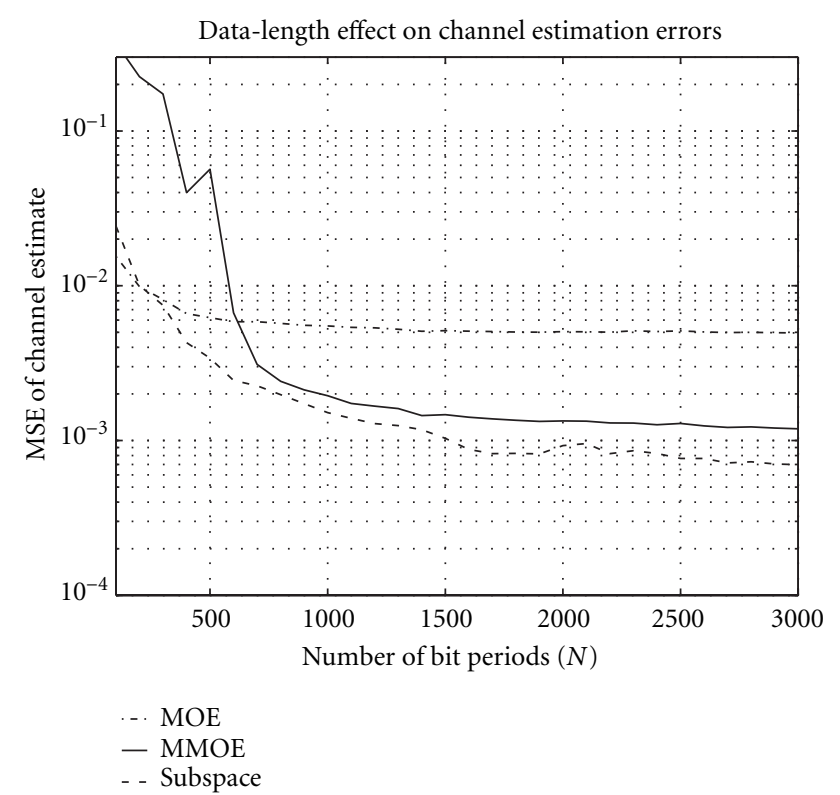

Figure 1: Comparison of data-length effect on the channel estimation error for different methods.

channel vector is then normalized. Totally $10 \mathrm{~dB}$ AWGN is added to the input. Totally 100 independent realizations are performed to obtain the average results.

We test the performance of the proposed MMOE method in different scenarios and compare it with the MOE method and the subspace method. The data length effect in terms of the number of received data vectors $(N)$ is first examined. Figure 1 presents the MSE of channel estimation. The dashdotted line is for the MOE method, the solid line represents the proposed method with $\alpha=0.7$, and the dashed line is for the subspace method. It can be seen that for a large $N$, the MMOE method is much better than the MOE method, and is close to the subspace method. Therefore, modification of the MOE optimization criterion leads to improvement in channel estimation. With these estimated channel parameters, the corresponding receivers exhibit different output SINRs, as shown in Figure 2. It is clear that the MMOE receiver gives higher SINRs than the MOE receiver and similar SINRs as the blind MMSE receiver.

Next, we test the noise effect when the input SNR varies from $0 \mathrm{~dB}$ to $50 \mathrm{~dB}$. In Figure 3, we compare the experimental results of channel estimation errors for the MMOE method (solid line) and the MOE method (dash-dotted line) with corresponding analytical results based on (24) with $\alpha=0.7$ $(0$ 's) and (25) (X's), respectively. It can be observed that the proposed method always outperforms the MOE method by almost $10 \mathrm{~dB}$. This difference agrees with the factor $(1-\alpha)^{2}$ in a logarithm scale as predicted by (24) and (25). It is also clear that the experimental results are highly consistent with the analytical results at high SNR upon which our approximation is based. Similarly, the output SINRs of the MMOE receiver and the MOE receiver are compared with the MMSE receiver and the SINR ratios plotted in

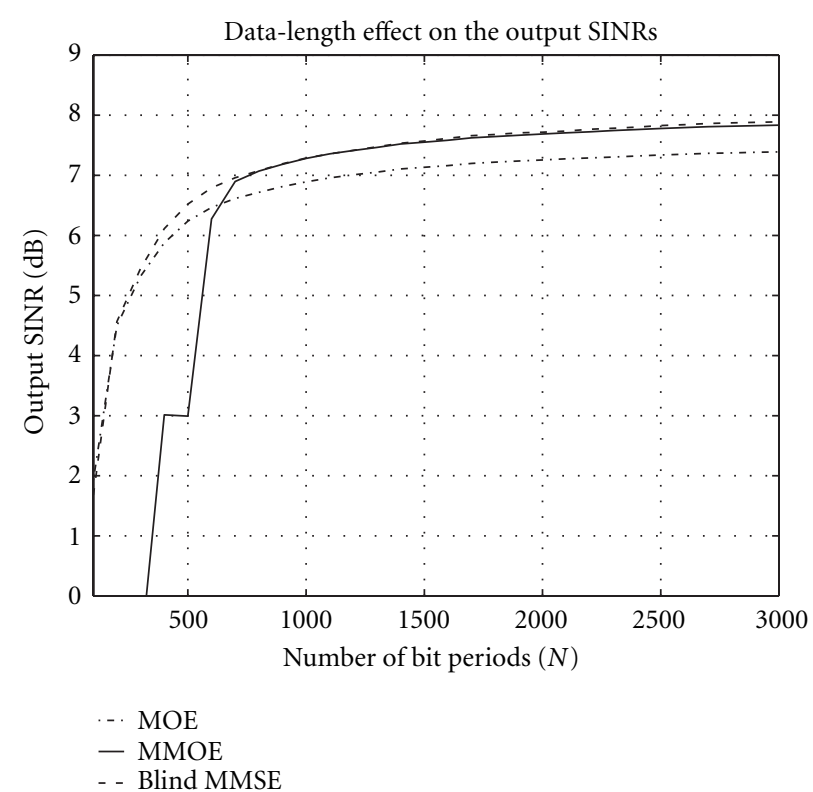

FIGURE 2: Comparison of data-length effect on the output SINR for different methods.

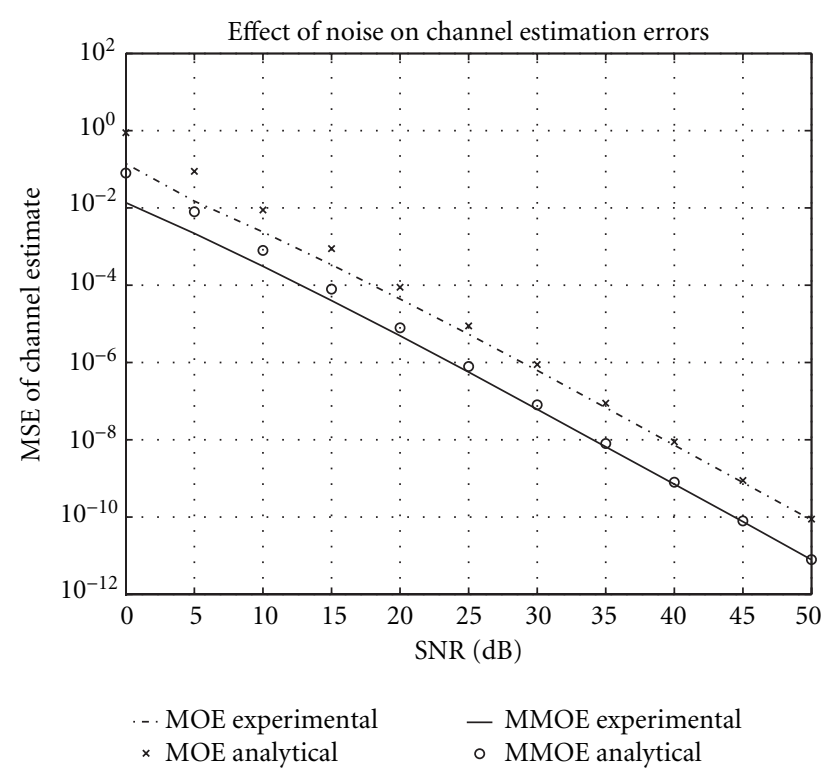

FIGURE 3: Channel estimation improvement of the MMOE method over the MOE method for different SNRs.

Figure 4. The analytical results are obtained from the reciprocals of (29). The solid line represents the experimental ratio $\operatorname{SINR}(\overline{\mathbf{g}}) / \mathrm{SINR}_{\mathrm{mmse}}$ of the proposed MMOE method, and $X$ 's represent its convergence level $1 /(1+\bar{\eta})$. The dash-dotted line is the experimental ratio $\operatorname{SINR}(\mathbf{g}) / \operatorname{SINR}_{\mathrm{mmse}}$ of the MOE method, and $\times$ 's stand for its convergence level $1 /(1+\eta)$. The consistency between the experimental result and the analytical result for each method is obvious when SNR is high. However, the difference between the MMOE method and the MOE method is evident. 


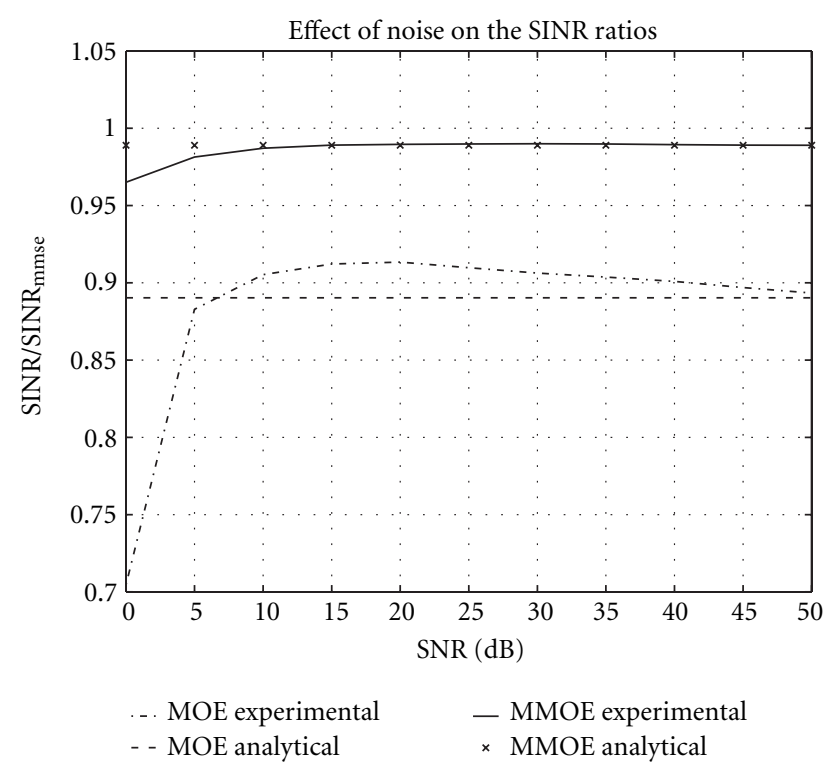

FIGURE 4: SINR improvement of the MMOE receiver over the MOE receiver for different SNRs.

It has been shown analytically that the parameter $\alpha$ has a significant effect on the performance of the MMOE method. When $\alpha=0$, the MMOE method degrades to the MOE method. When $\alpha \rightarrow 1^{-}$, the MMOE method approaches the subspace method if channel estimation is of our interest, or the MMOE receiver becomes the MMSE receiver if detection is performed. These claims can be verified by our simulation results for the channel estimation error in Figure 5 and the output SINR in Figure 6. SNR is still set to be $10 \mathrm{~dB}$. Parameter $\alpha$ varies from 0 to 1 . Around $\alpha=1$, we set $\alpha=0.999$ to approximate 1 in order for the required matrix to be invertible. In Figure 5, the experimental result from the MOE method at $\alpha=0$ coincides with the experimental result for the MMOE method as the dash-dotted line overlaps with the solid line. As $\alpha$ increases, the difference between these two methods increases, especially when $\alpha$ approaches 1 . Again, the analytical result agrees with the experimental result for the MMOE method. Figure 6 clearly shows the relationship among the MMOE receiver, the MOE receiver, and the MMSE receiver. It thus suggests that $\alpha$ should be selected to be as close to 1 as possible.

Performance loss from imperfect covariance estimation is further investigated for the MOE method. The effect of $N$ is tested for a range from 100 to $10^{4}$ and corresponding SINRs are shown in Figure 7 with SNR $=10 \mathrm{~dB}$. The dashed line represents the theoretical value (limit) as $N \rightarrow \infty$, dasheddotted line is from our analysis by (41), and solid line is based on simulation. It is observed that when $N$ reaches $2 \times 10^{3}$, the simulation result converges to the analytical one. SINR convergence is due to the convergence of the constraint vector to its optimal solution, as plotted in Figure 8, in terms of the MSE. The dashed line is our analytical result in (36) while the solid line is from our experiment. The point where the solid

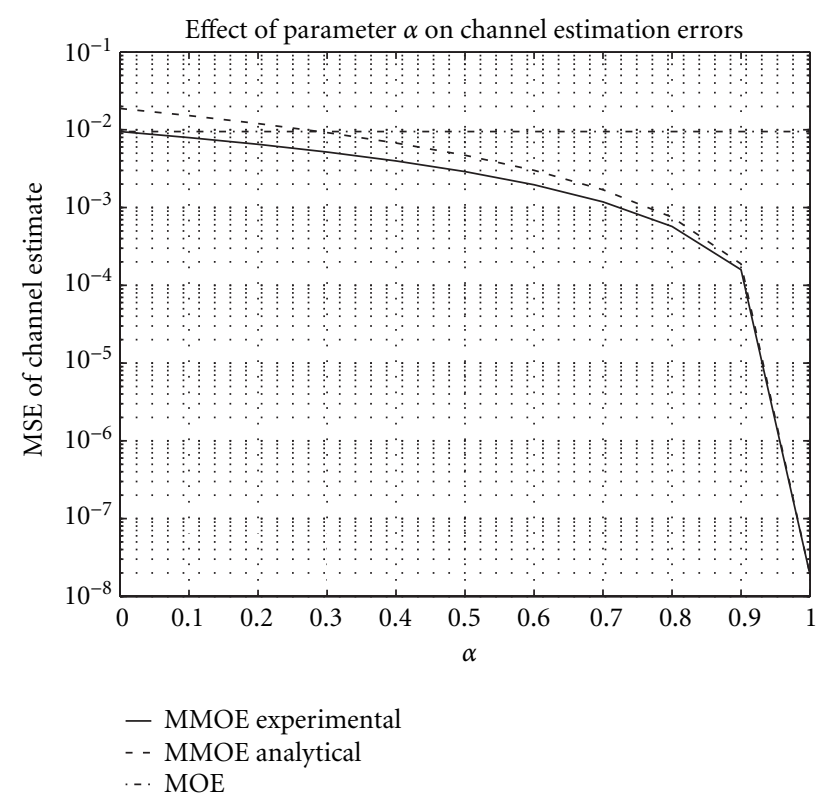

FIGURE 5: Effect of parameter $\alpha$ on the channel estimation error for the MMOE method.

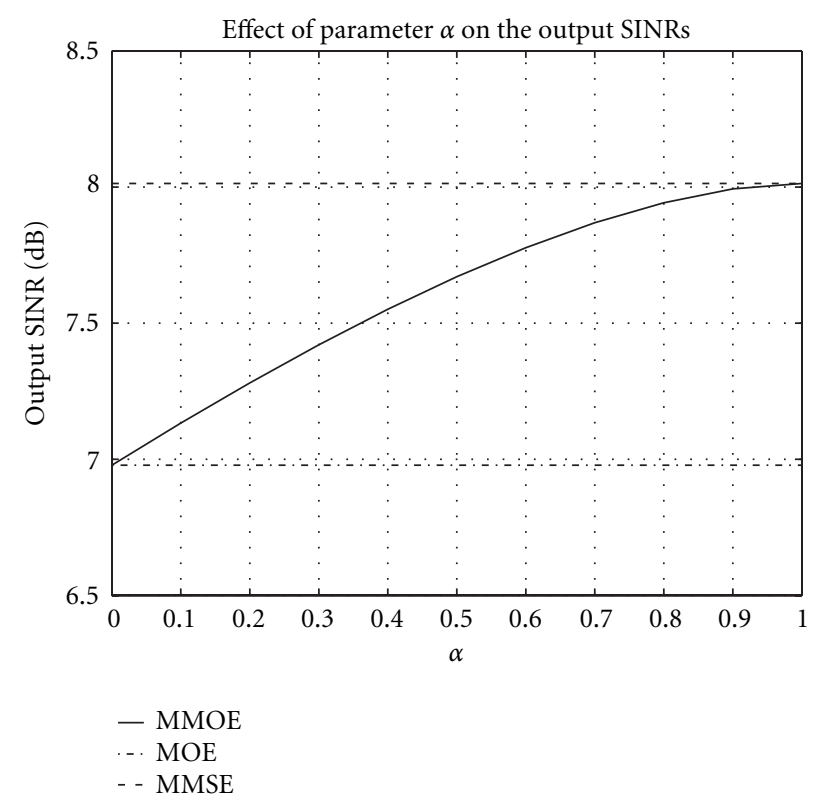

FIGURE 6: Effect of parameter $\alpha$ on the output SINR of the MMOE receiver.

line converges to the dashed line is approximately the same as that in Figure 5.

\section{CONCLUSIONS}

This paper provides detailed study on the MOE method when perfect estimation of the data covariance matrix is impossible. The performance of the MOE receiver, in terms of 


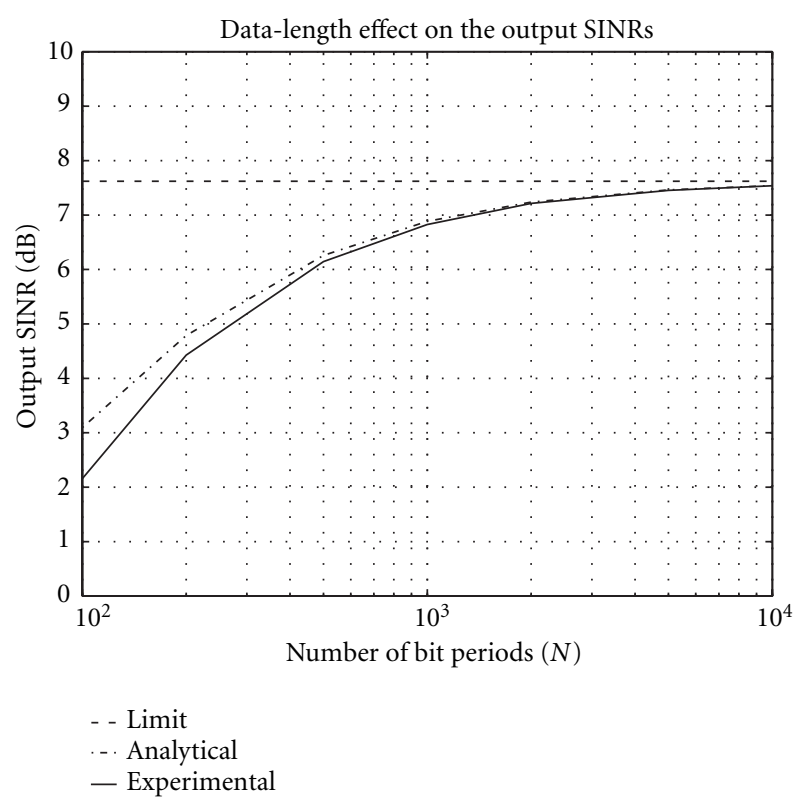

FIGURE 7: Data-length effect on the output SINR of the MOE receiver.

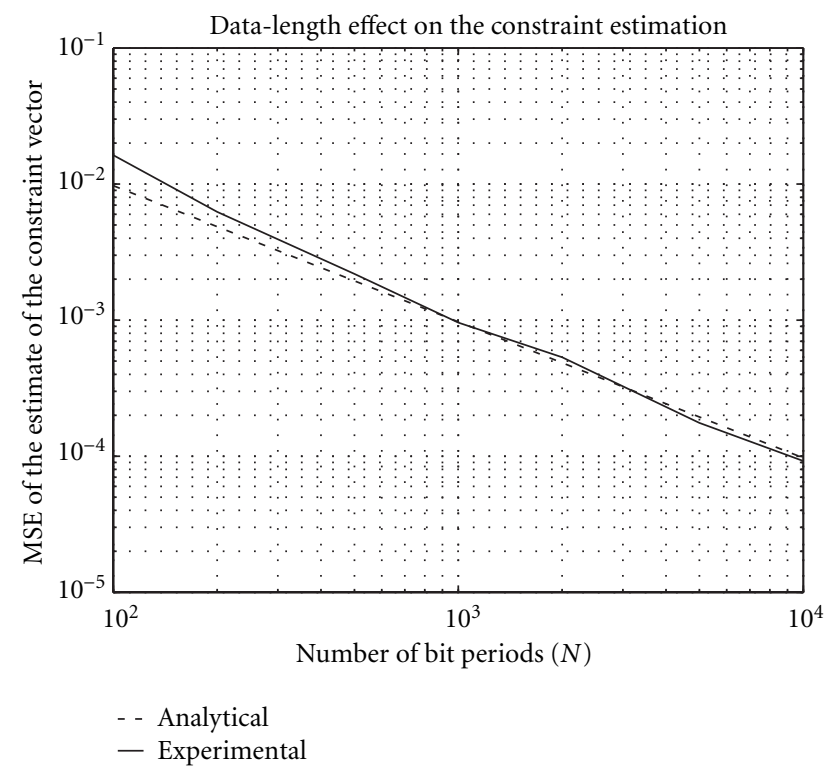

FIGURE 8: Data-length effect on the constraint estimation of the MOE method.

the output SINRs with respect to the number of received data vectors, is examined both analytically and experimentally. Due to the additive noise, the MOE receiver suffers from performance degradation compared with the blind MMSE receiver. Therefore, a modified MOE (MMOE) method is proposed by introducing a parameter $\alpha$ in the MOE constrained optimization criterion whose effect is throughly investigated. It is shown that significant improvement is achievable by properly selecting this parameter. It is also demonstrated that the MMOE method is closely related to the subspace method and exhibits agreement when $\alpha \rightarrow 1^{-}$. In such a scenario, the MMOE receiver also converges to the MMSE receiver.

\section{APPENDICES}

\section{A. PROOF OF PROPOSITION 3}

The SINRs of the MMSE receiver and the MOE receiver in (26) and (27) are direct results from [15, Appendix B]. Now, we derive (28) in details.

According to our input/output data model (5), the output SINR of our modified MOE receiver $\overline{\mathbf{f}}_{0}$ is given by the ratio of the signal power to the interference plus noise power

$$
\overline{\operatorname{SINR}}_{\text {mmoe }}=\frac{\sigma_{w_{1}}^{2} \overline{\mathbf{f}}_{0}^{H} \mathbf{h}_{1} \mathbf{h}_{1}^{H} \overline{\mathbf{f}}_{0}}{\overline{\mathbf{f}}_{0}^{H} \mathbf{R} \overline{\mathbf{f}}_{0}-\sigma_{w_{1}}^{2} \overline{\mathbf{f}}_{0}^{H} \mathbf{h}_{1} \mathbf{h}_{1}^{H} \overline{\mathbf{f}}_{0}},
$$

where $\overline{\mathbf{f}}_{0}^{H} \mathbf{R} \overline{\mathbf{f}}_{0}$ is the total output power. Substituting (16) into (A.1), we relate $\overline{\mathrm{SINR}}_{\mathrm{mmoe}}$ to the optimal constraint vector as follows:

$$
\overline{\mathrm{SINR}}_{\mathrm{mmoe}}=\frac{\sigma_{w_{1}}^{2} \overline{\mathbf{g}}_{0}^{H} \mathbf{C}^{H} \mathbf{R}^{-1} \mathbf{C} \mathbf{g}_{1} \mathbf{g}_{1}^{H} \mathbf{C}^{H} \mathbf{R}^{-1} \mathbf{C} \overline{\mathbf{g}}_{0}}{\overline{\mathbf{g}}_{0}^{H} \mathbf{C}^{H} \mathbf{R}^{-1} \mathbf{C} \overline{\mathbf{g}}_{0}-\sigma_{w_{1}}^{2} \overline{\mathbf{g}}_{0}^{H} \mathbf{C}^{H} \mathbf{R}^{-1} \mathbf{C} \mathbf{g}_{1} \mathbf{g}_{1}^{H} \mathbf{C}^{H} \mathbf{R}^{-1} \mathbf{C} \overline{\mathbf{g}}_{0}} .
$$

Based on (19) and (22) and noticing that $\mathbf{V}_{n}^{H} \mathbf{C g}_{1}=\mathbf{0}$, we have

$$
\begin{aligned}
\overline{\mathbf{g}}_{0}^{H} \mathbf{C}^{H} \mathbf{R}^{-1} \mathbf{C} \mathbf{g}_{1}= & \mathbf{g}_{1}^{H} \mathbf{A}_{1} \mathbf{g}_{1}-\beta \mathbf{g}_{1}^{H} \mathbf{A}_{1} \mathbf{A}_{0}^{\dagger} \mathbf{A}_{1} \mathbf{g}_{1} \\
& -\sigma_{v}^{2} \overline{\mathbf{g}}_{0}^{H} \mathbf{A}_{2} \mathbf{g}_{1}+O\left(\sigma_{v}^{4}\right)
\end{aligned}
$$

where the fact that $\beta=(1-\alpha) \sigma_{v}^{2}$ is in the order of $\sigma_{v}^{2}$ has been employed. Using (23) and noticing that $\mathbf{g}_{1}^{H} \mathbf{C}^{H} \mathbf{R}^{-1} \mathbf{C} \overline{\mathbf{g}}_{0}$ is the Hermitian of (A.3), we then obtain the quantity in the numerator of $\overline{\mathrm{SINR}}_{\mathrm{mmoe}}$

$$
\begin{aligned}
\overline{\mathbf{g}}_{0}^{H} \mathbf{C}^{H} \mathbf{R}^{-1} \mathbf{C g}_{1} \mathbf{g}_{1}^{H} \mathbf{C}^{H} \mathbf{R}^{-1} \mathbf{C} \overline{\mathbf{g}}_{0}= & \frac{1}{\sigma_{w_{1}}^{4}}-\frac{2 \beta}{\sigma_{w_{1}}^{2}} \mathbf{g}_{1}^{H} \mathbf{A}_{1} \mathbf{A}_{0}^{\dagger} \mathbf{A}_{1} \mathbf{g}_{1} \\
& -\frac{2 \sigma_{v}^{2}}{\sigma_{w_{1}}^{2}} \mathbf{g}_{1}^{H} \mathbf{A}_{2} \mathbf{g}_{1}+O\left(\sigma_{v}^{4}\right)
\end{aligned}
$$

Similarly, according to (19) and (22) and noticing that $\mathbf{V}_{n}^{H} \mathbf{C g}_{1}=\mathbf{0}$, we can find the first term in the denominator of $\overline{\mathrm{SINR}}_{\text {mmoe }}$,

$$
\begin{aligned}
\overline{\mathbf{g}}_{0}^{H} \mathbf{C}^{H} \mathbf{R}^{-1} \mathbf{C} \overline{\mathbf{g}}_{0}= & \mathbf{g}_{1}^{H} \mathbf{A}_{1} \mathbf{g}_{1}+\left(\frac{\beta^{2}}{\sigma_{v}^{2}}-2 \beta\right) \mathbf{g}_{1}^{H} \mathbf{A}_{1} \mathbf{A}_{0}^{\dagger} \mathbf{A}_{1} \mathbf{g}_{1} \\
& -\sigma_{v}^{2} \mathbf{g}_{1}^{H} \mathbf{A}_{2} \mathbf{g}_{1}+O\left(\sigma_{v}^{4}\right) .
\end{aligned}
$$

After considering (23), (A.5) becomes

$$
\begin{aligned}
\overline{\mathbf{g}}_{0}^{H} \mathbf{C}^{H} \mathbf{R}^{-1} \mathbf{C} \overline{\mathbf{g}}_{0}= & \frac{1}{\sigma_{w_{1}}^{2}}+\left(\frac{\beta^{2}}{\sigma_{v}^{2}}-2 \beta\right) \mathbf{g}_{1}^{H} \mathbf{A}_{1} \mathbf{A}_{0}^{\dagger} \mathbf{A}_{1} \mathbf{g}_{1} \\
& -\sigma_{v}^{2} \mathbf{g}_{1}^{H} \mathbf{A}_{2} \mathbf{g}_{1}+O\left(\sigma_{v}^{4}\right)
\end{aligned}
$$


Substituting (A.4) and (A.6) into (A.2), we have

$\overline{\operatorname{SINR}}_{\mathrm{mmoe}}=\frac{1+O\left(\sigma_{v}^{2}\right)}{\sigma_{v}^{2} \sigma_{w_{1}}^{2} \mathbf{g}_{1}^{H} \mathbf{A}_{2} \mathbf{g}_{1}+\left(\beta^{2} / \sigma_{v}^{2}\right) \sigma_{w_{1}}^{2} \mathbf{g}_{1}^{H} \mathbf{A}_{1} \mathbf{A}_{0}^{\dagger} \mathbf{A}_{1} \mathbf{g}_{1}+O\left(\sigma_{v}^{4}\right)}$.

After replacing $\beta$ by $(1-\alpha) \sigma_{v}^{2}$, (28) follows.

\section{B. DERIVATION OF MATRIX B}

For notational convenience, denote $\mathbf{y}(n)$ in (5) by $\mathbf{y}_{n}$, and $\mathbf{v}(n)$ by $\mathbf{v}_{n}$. We rewrite (5) in another form

$$
\mathbf{y}_{n}=\mathbf{H w}_{n}+\mathbf{v}_{n}
$$

where

$$
\mathbf{H}=\left[\mathbf{h}_{1}, \mathbf{H}_{i}\right], \quad \mathbf{w}_{n}=\left[w_{1}(n), \mathbf{w}_{i}^{T}(n)\right]^{T} .
$$

Substituting $\delta \mathbf{R}$ by $\widetilde{\mathbf{R}}-\mathbf{R}$, we obtain

$$
\mathbf{B}=E\{\tilde{\mathbf{R}} \mathbf{D} \tilde{\mathbf{R}}\}-\mathbf{R D R} .
$$

Assume $N$ data vectors are mutually independent for our derivation purpose. It can always be made possible by taking only those $N$ vectors which are not contributed by common inputs, although data vectors consecutive in time may be dependent on each other due to channel span. Then

$$
E\{\widetilde{\mathbf{R}} \widetilde{\mathbf{R}}\}=\frac{1}{N} \mathbf{C}_{1}+\left(1-\frac{1}{N}\right) \mathbf{R D R},
$$

where $\mathbf{C}_{1} \triangleq E\left\{\mathbf{y}_{n} \mathbf{y}_{n}^{H} \mathbf{D} \mathbf{y}_{n} \mathbf{y}_{n}^{H}\right\}$. Therefore,

$$
\mathbf{B}=\frac{1}{N}\left(\mathbf{C}_{1}-\mathbf{R D R}\right) .
$$

According to (B.1), $\mathbf{y}_{n} \mathbf{y}_{n}^{H}$ can be expanded into four terms

$$
\mathbf{y}_{n} \mathbf{y}_{n}^{H}=\mathbf{H} \mathbf{w}_{n} \mathbf{w}_{n}^{H} \mathbf{H}^{H}+\mathbf{H} \mathbf{w}_{n} \mathbf{v}_{n}^{H}+\mathbf{v}_{n} \mathbf{w}_{n}^{H} \mathbf{H}^{H}+\mathbf{v}_{n} \mathbf{v}_{n}^{H} .
$$

The noise $\mathbf{v}_{n}$ has been assumed to be zero-mean white Gaussian. It is independent of the input sequence which is i.i.d. with zero-mean, variance $\boldsymbol{\Gamma}$, and finite fourth-order moment. Since $E\left\{\mathbf{v}_{n} \mathbf{v}_{n}^{H}\right\}=\sigma_{v}^{2} \mathbf{I}, E\left\{\mathbf{w}_{n} \mathbf{w}_{n}^{H}\right\}=\boldsymbol{\Gamma}$. Only the following terms survive in $\mathbf{C}_{1}$,

$$
\begin{aligned}
\mathbf{C}_{1}= & \mathbf{H C}_{2} \mathbf{H}^{H}+\mathbf{C}_{3}+\mathbf{H} E\left\{\mathbf{w}_{n} \mathbf{w}_{n}^{T}\right\} \mathbf{H}^{T} \mathbf{D}^{T} E\left\{\mathbf{v}_{n}^{*} \mathbf{v}_{n}^{H}\right\} \\
& +E\left\{\mathbf{v}_{n} \mathbf{v}_{n}^{T}\right\} \mathbf{D}^{T} \mathbf{H}^{*} E\left\{\mathbf{w}_{n}^{*} \mathbf{w}_{n}^{H}\right\} \mathbf{H}^{H}+\sigma_{v}^{2} \operatorname{tr}(\mathbf{D}) \mathbf{H} \boldsymbol{\Gamma} \mathbf{H}^{H} \\
& +\sigma_{v}^{2} \operatorname{tr}\left(\mathbf{D H} \mathbf{H} \mathbf{H}^{H}\right) \mathbf{I}+\sigma_{v}^{2} \mathbf{H} \boldsymbol{\Gamma} \mathbf{H}^{H} \mathbf{D}+\sigma_{v}^{2} \mathbf{D} \mathbf{H} \boldsymbol{\Gamma} \mathbf{H}^{H},
\end{aligned}
$$

where tr denotes the trace of a matrix, superscript “*” denotes complex conjugate

$$
\begin{aligned}
& \mathbf{C}_{2} \triangleq E\left\{\mathbf{w}_{n} \mathbf{w}_{n}^{H} \mathbf{H}^{H} \mathbf{D} \mathbf{H} \mathbf{w}_{n} \mathbf{w}_{n}^{H}\right\}, \\
& \mathbf{C}_{3} \triangleq E\left\{\mathbf{v}_{n} \mathbf{v}_{n}^{H} \mathbf{D} \mathbf{v}_{n} \mathbf{v}_{n}^{H}\right\} .
\end{aligned}
$$

The last four terms in $\mathbf{C}_{1}$ are deterministic quantities. The first four terms are dependent on the statistical properties of the transmitted signal and the noise. Here as an example, we restrict our attention to a typical scenario: the transmitted symbols from different users are taken from real finite alphabets and have equal power, and the noise is also real. For all other cases, we can follow the similar procedures detailed next. However, we will not enumerate them for our concise presentation.

We denote the variance of the input by $\sigma_{w}^{2}$ and its fourth order moment by $m_{4 w}$. Then

$$
\boldsymbol{\Gamma}=E\left\{\mathbf{w}_{n} \mathbf{w}_{n}^{T}\right\}=\sigma_{w}^{2} \mathbf{I}, \quad E\left\{\mathbf{v}_{n} \mathbf{v}_{n}^{T}\right\}=\sigma_{v}^{2} \mathbf{I} .
$$

To easily evaluate $\mathbf{C}_{2}$ and $\mathbf{C}_{3}$, we perform vec operations first to combine corresponding terms, similarly as [21]. Then reverse operations unvec are applied to obtain these matrices

$$
\begin{aligned}
& \mathbf{C}_{2}=\operatorname{unvec}\left[\mathbf{C}_{4} \operatorname{vec}\left(\mathbf{H}^{H} \mathbf{D H}\right)\right], \\
& \mathbf{C}_{3}=\operatorname{unvec}\left[\mathbf{C}_{5} \operatorname{vec}(\mathbf{D})\right],
\end{aligned}
$$

where the property of vec has been applied [22], and

$$
\begin{aligned}
& \mathbf{C}_{4} \triangleq E\left\{\left(\mathbf{w}_{n} \mathbf{w}_{n}^{T}\right) \otimes\left(\mathbf{w}_{n} \mathbf{w}_{n}^{T}\right)\right\}, \\
& \mathbf{C}_{5} \triangleq E\left\{\left(\mathbf{v}_{n} \mathbf{v}_{n}^{T}\right) \otimes\left(\mathbf{v}_{n} \mathbf{v}_{n}^{T}\right)\right\} .
\end{aligned}
$$

Then it can be verified that [21]

$$
\mathbf{C}_{4}=\left(m_{4 w}-3 \sigma_{w}^{4}\right) \mathbf{X}_{1}+\sigma_{w}^{4} \mathbf{X}_{2}+\sigma_{w}^{4} \mathbf{I},
$$

where

$$
\begin{aligned}
& \mathbf{X}_{1}=\operatorname{diag}\left\{\mathbf{a}_{1} \mathbf{a}_{1}^{T}, \ldots, \mathbf{a}_{L} \mathbf{a}_{L}^{T}\right\}, \\
& \mathbf{a}_{l}^{T}=[0, \ldots, 0, \underbrace{1}_{\text {lth element }}, 0, \ldots, 0]_{1 \times L}, \\
& \mathbf{X}_{2}=\left[\tilde{\mathbf{X}}_{i, j}\right]_{L \times L}, \quad \tilde{\mathbf{X}}_{i, j}=\mathbf{a}_{i} \mathbf{a}_{j}^{T}+\mathbf{a}_{j} \mathbf{a}_{i}^{T},
\end{aligned}
$$

and $L$ is the length of the input vector $\mathbf{w}_{n}$. Similarly, we can evaluate $\mathbf{C}_{5}$. Since the second-order and fourth-order moments of a white Gaussian process are related, we obtain

$$
\mathbf{C}_{5}=\sigma_{v}^{4} \mathbf{X}_{3}+\sigma_{v}^{4} \mathbf{I},
$$

where

$$
\begin{aligned}
\mathbf{X}_{3} & =\left[\tilde{\widetilde{\mathbf{X}}}_{i, j}\right]_{P \times P}, \quad \tilde{\widetilde{\mathbf{X}}}_{i, j}=\mathbf{b}_{i} \mathbf{b}_{j}^{T}+\mathbf{b}_{j} \mathbf{b}_{i}^{T}, \\
\mathbf{b}_{l}^{T} & =[0, \ldots, 0, \underbrace{1}_{\text {lth element }}, 0, \ldots, 0]_{l \times P},
\end{aligned}
$$

and $P$ is the length of the data vector (or the noise vector $\mathbf{v}_{n}$ ). Substituting (B.13) in (B.10) and (B.15) in (B.11), respectively, we obtain

$$
\begin{aligned}
& \mathbf{C}_{2}=\mathbf{C}_{6}+\sigma_{w}^{4} \mathbf{H}^{H} \mathbf{D H}, \\
& \mathbf{C}_{3}=\operatorname{unvec}\left[\sigma_{v}^{4} \mathbf{X}_{3} \operatorname{vec}(\mathbf{D})\right]+\sigma_{v}^{4} \mathbf{D},
\end{aligned}
$$


where

$$
\mathbf{C}_{6}=\text { unvec }\left\{\left[\left(m_{4 w}-3 \sigma_{w}^{4}\right) \mathbf{X}_{1}+\sigma_{w}^{4} \mathbf{X}_{2}\right] \operatorname{vec}\left(\mathbf{H}^{H} \mathbf{D H}\right)\right\} .
$$

Observe that

$$
\mathbf{R}=\sigma_{w}^{2} \mathbf{H H}^{T}+\sigma_{v}^{2} \mathbf{I}
$$

Substituting (B.9) and (B.17) in (B.7) first, then (B.7) in (B.5), we obtain

$$
\begin{aligned}
\mathbf{B}= & \frac{\sigma_{w}^{2} \sigma_{v}^{2}}{N}\left(\mathbf{H H}^{T} \mathbf{D}^{T}+\mathbf{D}^{T} \mathbf{H}^{*} \mathbf{H}^{H}\right) \\
& +\frac{\sigma_{w}^{2} \sigma_{v}^{2}}{N}\left[\operatorname{tr}(\mathbf{D}) \mathbf{H} \mathbf{H}^{H}+\operatorname{tr}\left(\mathbf{D H} \mathbf{H}^{H}\right) \mathbf{I}\right] \\
& +\frac{\sigma_{v}^{4}}{N} \operatorname{unvec}\left[\mathbf{X}_{3} \operatorname{vec}(\mathbf{D})\right]+\frac{1}{N} \mathbf{H C}_{6} \mathbf{H}^{H},
\end{aligned}
$$

which is our desired result.

\section{ACKNOWLEDGMENT}

This paper was presented in part at the 35th Asilomar Conference on Signals, Systems, and Computers, Pacific Grove, Calif, November 2001.

\section{REFERENCES}

[1] B. Arroyo-Fernandez, J. Dasilva, J. Fernandes, and R. Prasad, "Life after third-generation mobile communications," IEEE Communications Magazine, vol. 39, no. 8, pp. 41-42, 2001.

[2] W. Lu, "Broadband wireless access technologies and applications," IEEE Communications Magazine, vol. 39, no. 9, pp. 111-145, 2001.

[3] G. Cherubini, J. M. Cioffi, A. Duel-Hallen, and H. V. Poor, "Multiuser detection techniques with application to wired and wireless communications systems I," IEEE Journal on Selected Areas in Communications, vol. 19, no. 8, August 2001.

[4] U. Mitra, "Comparison of maximum-likelihood-based detection for two multirate access schemes for CDMA signals," IEEE Trans. Communications, vol. 47, no. 1, pp. 64-77, 1999.

[5] H. V. Poor, "Active interference suppression in CDMA overlay systems," IEEE Journal on Selected Areas in Communications, vol. 19, no. 1, pp. 4-20, 2001.

[6] S. Verdú, Multiuser Detection, Cambridge University Press, New York, NY, USA, 1998.

[7] S. Verdú, "Minimum probability of error for asynchronous Gaussian multiple-access channels," IEEE Transactions on Information Theory, vol. 32, no. 1, pp. 85-96, 1986.

[8] M. Honig, U. Madhow, and S. Verdú, "Blind adaptive multiuser detection," IEEE Transactions on Information Theory, vol. 41, no. 4, pp. 944-960, 1995.

[9] D. Gesbert, J. Sorelius, P. Stoica, and A. Paulraj, "Blind multiuser MMSE detector for CDMA signals in ISI channels," IEEE Communications Letters, vol. 3, no. 8, pp. 233-235, 1999.

[10] S. Bensley and B. Aazhang, "Subspace-based channel estimation for code division multiple access communication systems," IEEE Trans. Communications, vol. 44, no. 8, pp. 10091020, 1996.
[11] M. Torlak and G. Xu, "Blind multiuser channel estimation in asynchronous CDMA systems," IEEE Trans. Signal Processing, vol. 45, no. 1, pp. 137-147, 1997.

[12] X. Wang and H. V. Poor, "Blind multiuser detection: a subspace approach," IEEE Transactions on Information Theory, vol. 44, no. 2, pp. 677-690, 1998.

[13] X. Wang and H. V. Poor, "Blind equalization and multiuser detection in dispersive CDMA channels," IEEE Trans. Communications, vol. 46, no. 1, pp. 91-103, 1998.

[14] H. Liu and K. Li, "A decorrelating RAKE receivers for CDMA communications over frequency-selective fading channels," IEEE Trans. Communications, vol. 47, no. 7, pp. 1036-1045, 1999.

[15] M. Tsatsanis and Z. Xu, "Performance analysis of minimum variance CDMA receivers," IEEE Trans. Signal Processing, vol. 46, no. 11, pp. 3014-3022, 1998.

[16] Z. Tian, K. Bell, and H. Van Trees, "Robust constrained linear receivers for CDMA wireless systems," IEEE Trans. Signal Processing, vol. 49, no. 7, pp. 1510-1522, 2001.

[17] Z. Xu and M. Tsatsanis, "Blind adaptive algorithms for minimum variance CDMA receivers," IEEE Trans. Communications, vol. 49, no. 1, pp. 180-194, 2001.

[18] Z. Xu, "Perturbation study on MOE-based multiuser detection," in Proc. 35th Asilomar Conf. on Signals, Systems, and Computers (Asilomar '01), vol. 2, pp. 1733-1737, Pacific Grove, Calif, USA, November 2001.

[19] B. Friedlander and A. Weiss, "On the second-order statistics of the eigenvectors of sample covariance matrices," IEEE Trans. Signal Processing, vol. 46, no. 11, pp. 3136-3139, 1998.

[20] F. Li, H. Liu, and R. Vaccaro, "Performance analysis for DOA estimation algorithms: Further unification, simplification, and observations," IEEE Trans. on Aerospace and Electronics Systems, vol. 29, no. 4, pp. 1170-1184, 1993.

[21] Z. Xu, "Asymptotically near-optimal blind estimation of multipath CDMA channels," IEEE Trans. Signal Processing, vol. 49, no. 9, pp. 2003-2017, 2001.

[22] P. Lancaster and M. Tismenetsky, The Theory of Matrices, Academic Press, San Diego, Calif, USA, 2nd edition, 1985.

Zhengyuan $\mathrm{Xu}$ received both the B.E. and M.E. degrees in electronic engineering from Tsinghua University, Beijing, China, in 1989 and 1991, respectively, and the Ph.D. degree in electrical engineering from Stevens Institute of Technology, Hoboken, NJ, in 1999. From 1991 to 1996 , he worked as an Engineer and Department Manager at the Tsinghua Unisplendour Group Corp., Tsinghua University. From 1996 to 1999, he was a Research Assistant and Research Associate at Stevens Institute of Technology, working on signal processing for wireless communications, especially for multiuser CDMA systems. In 1999, he joined the Department of Electrical Engineering, University of California, Riverside, as an Assistant Professor. His current research interests include advanced signal processing, multirate communication, multiuser detection, and system identification. Dr. Xu received the Outstanding Student Award and the Motorola Scholarship from Tsinghua University, and the Peskin Award from Stevens Institute of Technology. He also received the Academic Senate Research Award and the Regents' Faculty Award from University of California, Riverside. He is an IEEE senior member. 